\title{
The Radiometric Measurement Quantity for SAR Images
}

\author{
Björn J. Döring and Marco Schwerdt
}

\begin{abstract}
A Synthetic Aperture Radar (SAR) system measures among other quantities the terrain radar reflectivity. After image calibration, the pixel intensities are commonly expressed in terms of radar cross sections (for point targets) or as backscatter coefficients (for distributed targets), which are directly related. This paper argues that pixel intensities are not generally proportional to radar cross section or derived physical quantities. The paper further proposes to replace the inaccurate term radar cross section by equivalent radar cross section as the radiometric measurement quantity for SAR images. The measurement procedure as such remains unchanged.

Two problems are seen with using radar cross section as the radiometric measurement quantity for point targets. First, the radar cross section is frequency and/or angular dependent for many targets, whereas a SAR system operates not at a single set but through a range of frequencies and aspect angles. This begs the question of which radar cross section within this twodimensional range should actually be taken as the measurement result. Second, the pixel intensities seen in SAR images are the output of a filtering process, which is affected by the complex transfer function of the point target. Radar cross section, on the other hand, does not depend on phase.

The equivalent radar cross section expresses the reflectivity in terms of the radar cross section of an equivalent conducting sphere which would result in the same pixel intensity as the one observed if the sphere were to replace the actual target in the scene. This distinction is crucial in defining the measurand for radiometric SAR measurements in principal, and hence it is important for radiometric SAR calibration. It is furthermore of particular practical importance for current and emerging wideband, high-resolution, and high-accuracy SAR systems.
\end{abstract}

\section{INTRODUCTION}

Synthetic Aperture Radar (SAR) images play a key role in many remote sensing applications. A SAR instrument, combined with data processing facilities, can be regarded as a measuring device. One of the measurement quantities of the SAR instrument is terrain radar reflectivity. This is then used as input data in numerous inverse problems to derive physical quantities such as soil moisture level, biomass, and salinity. It is therefore crucial to have a clear understanding of the radar observable, namely radar reflectivity.

The quantities directly measurable by a digital SAR system are amplitude and phase, recorded for each image element. After data processing, amplitude and phase remain to be calibrated. At this stage, the pixel magnitudes, and their equivalent pixel intensities, are expressed as uncalibrated digital numbers.

The known systematic radiometric offsets, described by a factor, are used to scale the pixel intensities during calibration,

The authors are with the Microwaves and Radar Institute of the German Aerospace Center (DLR), Oberpfaffenhofen, Germany.

Manuscript received Month DD, 2011; revised Month DD, 2012. a necessary prerequisite to yield comparable image products [1]. After radiometric calibration, the pixel quantities are commonly expressed in terms of the (specific) backscattering coefficient or similar quantities [1]-[4], which are directly related to the definition of Radar Cross Section (RCS) of point targets. In this paper it is argued that radar cross section is not the actual radiometric measurement quantity in SAR images. The underlying reason for this is the neglected spectral and angular-dependent reflectivity of most natural and manmade radar targets. The authors propose to use the terminology equivalent radar cross section instead.

Introducing this new terminology does not require a change of the radiometric measurement process as such. For instance, no new correction factors need to be introduced because especially the frequency dependent reflectivity of radar targets is a feature that a SAR system actually desires to, and already does, detect. Multispectral SAR systems, as a point in case, put a focus on taking advantage of the frequency dependent reflectivity. However, the new terminology changes the way in which radiometric SAR products should be annotated and calibrated. The distinction between RCS and equivalent RCS has special practical importance for wideband, high-resolution, and radiometrically accurate SAR systems for which the new terminology can resolve an inaccuracy in description.

The proposed changes for radiometric measurements as such are necessary but can be perceived as notational only. However, and this is the important innovation from a practical point of view, radiometric calibration with reference point targets needs to be adapted by introducing target-dependent and system-dependent corrections that result from the inherent spectral and angular dependence of reflectivity for every practical reference target.

The discussion in the following sections is concentrated on point targets (for which the measurement quantity is the (equivalent) radar cross section), and not on distributed targets (for which the measurement quantity is the (equivalent) backscatter coefficient or related quantities). This is without loss of generality because the determination of the (equivalent) backscatter coefficient for a distributed target depends on prior measurement of a point target with known reflectivity during radiometric calibration [1].

This paper initially identifies the problems that are associated with the term RCS as the measurement quantity in Sec. II. The following Sec. III introduces the new terminology to resolve the problems. Finally, Sec. IV and V discuss the implications of the proposed terminology for measurements and calibration, respectively. 


\section{Problem Description}

The fundamental problem of using RCS as the output measurement quantity for describing pixel intensities in SAR images is rooted in the underlying, often only implied, measurement model. In fact, the pixel intensities in SAR images are only accurately representative of the target RCS if the point target transfer function $H_{t}$ can be modeled as

$$
H_{t}(f, \boldsymbol{\alpha})=\text { const }
$$

within the relevant parameter range, where $f$ is frequency and $\boldsymbol{\alpha}=(\theta, \phi)$ describes the (monostatic) angle under which the target is seen by the radar. Whereas this provides a practical and justified approximation for many natural targets observed by a sufficiently narrow-band SAR system with sufficiently low azimuth resolution, it does not hold for all cases. Principally, natural as well as man-made radar targets have frequency and angular-dependent backscattering characteristics due to their electromagnetic properties, their size, and shape. Therefore, point targets should rather be modeled by

$$
H_{t}(f, \boldsymbol{\alpha})=A(f, \boldsymbol{\alpha}) \mathrm{e}^{j \varphi(f, \boldsymbol{\alpha})}
$$

where $A(f, \boldsymbol{\alpha})$ and $\varphi(f, \boldsymbol{\alpha})$ are the frequency and angulardependent gain and phase functions, respectively. The simplified measurement model in Eq. (1) is inherent within the more general definition, Eq. (2).

In the following paragraphs it shall be shown that the received SAR signal reflected by a point target, after pulse compression, is in fact not generally proportional to its radar cross section because the target backscattering depends, in principle, on frequency and angle.

In existing SAR literature, the relationship between the radar system, the point target, and the received signal is typically introduced by considering a monostatic real aperture radar system without a matched filter at the receiver. For these systems, the monostatic radar equation,

$$
P_{r}=\frac{P_{t} G^{2} \lambda^{2}}{(4 \pi)^{3} R^{4}} \sigma
$$

expresses the received power $P_{r}$ in terms of the transmitted power $P_{t}$, the receive and transmit antenna gain $G$, the wavelength $\lambda$, the radar-target distance $R$, and the point target radar cross section $\sigma$. The radar cross section $\sigma$ of any target, in this case the wanted measurand, is defined as the scaled ratio of the scattered power (seen at distance $R$ away from the point target) to the incident power. This can be written as

$$
\sigma=\lim _{R \rightarrow \infty} 4 \pi R^{2} \frac{\left|\mathbf{E}_{s}\right|^{2}}{\left|\mathbf{E}_{i}\right|^{2}},
$$

where $\mathbf{E}_{s}$ and $\mathbf{E}_{i}$ are the scattered and incident electrical fields [5].

Assuming for the moment no angular dependence of the target reflectivity, Eq. (3) can now be reformulated as

$$
P_{r}(f)=\frac{P_{t}(f) G^{2}(f) \lambda(f)^{2}}{(4 \pi)^{3} R^{4}} \sigma(f) .
$$

All parameters except $R$ depend on frequency $f$. This points toward the first dilemma: A SAR system uses the principle of matched filtering and pulse compression in order to increase the signal-to-noise ratio and range resolution. Therefore, the transmitted SAR signal covers a defined bandwidth; typically a linear frequency modulated pulse is used. If the power scattered back by the point target depends on frequency, then the question arises as to which RCS should actually be annotated in the SAR image. Certainly the RCS at the center frequency is only representative if the target RCS can be approximated to be constant over the relevant bandwidth according to Eq. (1), an assumption which is seldom explicitly stated in the literature. The arithmetic mean of the RCS over the pulse bandwidth also does not lead to the wanted result because receiver hardware and pulse compression introduce a frequency dependence produced by the receiver. In practice, the workaround of annotating a single backscatter coefficient per pixel in SAR products [6], [7] possibly erroneously implies a constant target RCS over the range frequency bandwidth. By doing this a measurement uncertainty is introduced due to the mathematical model of the measurand.

The previous discussion can likewise be extended to the azimuth direction. The azimuth resolution in SAR is achieved by coherent sampling of a point target's complex reflectivity under different aspect angles, and subsequent compression. If the target RCS significantly varies over the processed aspect angle range (as can be the case for dihedrals in urban environments, for instance), then again will the pixel intensity not be proportional to any particular RCS, but it will be proportional to a weighted average of the RCS over the angular range.

The initial dilemma arises for any radar system that does not measure the point target's radar cross section $\sigma(f, \boldsymbol{\alpha})$ at a single frequency or angle. The following problem arises for SAR systems when the transition is made from the raw to the processed data domain, which depends on complex reflectivity, i.e. magnitude and phase. The SAR receiver records the target data as complex amplitudes $S(x, y) ; x$ and $y$ are spatial coordinates. Neglecting noise, the complex SAR image is then formed by

$$
V(x, y)=\sqrt{K} S(x, y) * h(x, y),
$$

where $K$ is a complex, absolute calibration coefficient (known through calibration), $*$ denotes convolution, and $h$ is the SAR system point-spread function [1]. Conventionally, it is assumed that the raw data resulting from a single point target in the image can be described by

$$
S_{e}(x, y)=\sqrt{\sigma_{e}} \delta(x, y),
$$

where $\sigma_{e}$ is the point target's radar cross section ${ }^{1}$, and $\delta(x, y)$ is the Dirac delta function. This is equivalent to the target description in Eq. (1). The point target's RCS is derived from the square law detected complex image [1]

$$
P_{e}(x, y)=|V(x, y)|^{2}=K \sigma_{e}|\delta(x, y) * h(x, y)|^{2}
$$

by integration over a sufficiently large region $A$ (allowing the approximation $\left.\iint_{A} h(x, y) \mathrm{d} x \mathrm{~d} y \approx 1\right)$ [1], [8] so that the

\footnotetext{
${ }^{1}$ Later on, $\sigma_{e}$ will be called equivalent radar cross section. The equivalent radar cross section is identical to the radar cross section $\sigma$ for targets which can be described by Eq. (1), as is the case in this paragraph.
} 
integrated point target intensity $I_{e}$ becomes

$$
I_{e}=\iint_{A} P(x, y) \mathrm{d} x \mathrm{~d} y=K \sigma_{e} .
$$

The integrated pixel intensity is therefore directly proportional to the point target RCS for uniform targets according to Eq. (6). This summarizes the conventional understanding [1][4] of how pixel intensities are linked to RCS.

In contrast to the above derivation, a target with arbitrary frequency and angular dependence according to Eq. (2) shall now be considered. Equation (6) becomes

$$
S_{e}^{\prime}(x, y)=\sqrt{\sigma_{e}^{\prime}} h_{t n}(x, y)
$$

where $h_{t n}$ is the normalized point target's point-spread function $^{2}$ (see Sec. V for details), and $\sigma_{e}^{\prime}$ is a scalar scaling factor describing the magnitude of the reflectivity. Hence, the square law detected image becomes

$$
P_{e}^{\prime}(x, y)=K \sigma_{e}^{\prime}\left|h_{t n}(x, y) * h(x, y)\right|^{2},
$$

and the integrated intensity is

$$
\begin{aligned}
I_{e}^{\prime} & =\iint_{A} P(x, y) \mathrm{d} x \mathrm{~d} y \\
& =K \sigma_{e}^{\prime} \iint_{A}\left|h_{t n}(x, y) * h(x, y)\right|^{2} \mathrm{~d} x \mathrm{~d} y .
\end{aligned}
$$

The convolution operation effectively performs a weighted average of the point target's point-spread function over frequency and aspect angle. In contrast to Eqs. (7) and (8), the pixel intensities $P_{e}^{\prime}(x, y)$ and the integrated pixel intensity $I_{e}^{\prime}$ therefore do not only depend on the scaling factor $\sigma_{e}^{\prime}$ anymore, but also on the point target's complex transfer function.

The definition of RCS in Eq. (4) only considers a ratio of powers, i.e., magnitudes; the phase is irrelevant for its definition. The filtering operation in Eq. (10), on the other hand, operates on complex signals, and both the signal magnitude and phase over time influence the filter output. In conclusion, this discrepancy between the definition of RCS (incorporating absolute magnitudes) and filtering (depending on magnitude and phase) is the second reason why target RCS is unsuitable to describe the pixel intensities in SAR images. Two point targets with identical RCS can result in distinct integrated pixel intensities.

In conclusion, the SAR signal of a point target after pulse compression is only proportional to its RCS if its frequency and angle-dependent transfer function can be approximated as constant in amplitude and phase over the relevant frequency and angular range. In the general case, the integrated SAR pixel intensity after processing depends on the transfer function of the point target because the processing is a filtering process over the system bandwidth and aspect angle range.

\section{Proposed New Radiometric Measurement QUANTITY TERMINOLOGY}

It was argued that, in the general case, the pixel intensities in SAR images are not simply proportional to the target

\footnotetext{
${ }^{2}$ The dependence of the point spread function $h_{t n}$ on spatial coordinates $(x, y)$ can equivalently be expressed as a dependence on azimuth and range time $\left(t_{a}, t_{r}\right)$, or frequency and angle $(f, \boldsymbol{\alpha})$.
}

radar cross section $\sigma$, neither are derived quantities like the backscattering coefficient $\sigma^{0}$. In other words, the measurement quantity in radiometric SAR measurements is not RCS, and therefore it should not be referred to as RCS.

It is proposed to instead call the measurement quantity for point targets equivalent radar cross section. The equivalent radar cross section $\sigma_{e}$ shall be equal to the radar cross section of a perfectly conducting sphere which would result in an equivalent pixel intensity if the sphere were to replace the measured target. The definition exploits the frequency and angular independence of the RCS of a sphere with radius $a$

$$
\sigma_{\text {sphere }}=\pi a^{2}
$$

which is a valid approximation as long as the sphere circumference is much (greater than ten times) larger than the wavelength [5].

Replacing RCS by equivalent RCS pays tribute to the two general points of critique. Now,

- the filtering of complex signals according to Eq. (5) is correctly distinguished from the definition of RCS, which only takes signal magnitudes into consideration, and

- cases are covered for which the target's RCS dependence on frequency or angle are significant according to Eq. (2).

One could say that the terminology of equivalent RCS allows distinction between the target RCS (Eq. (4)) and the target's pixel intensity as seen through the eyes of the SAR processing filter (Eq. (10)). The proposed terminology is applicable to target backscatters with an arbitrary frequency and angular dependence according to Eq. (2), including targets with a flat frequency and angular response. Depending on the target, the measurement uncertainty can appear greatly reduced due to the more accurate measurement model, which especially benefits high bandwidth, high resolution systems requiring a high radiometric accuracy.

The transition from the present to the proposed terminology does not pose difficulties. For instance, the measurement unit for RCS and equivalent RCS is the same: square meter. Also, it is straightforward to transform the backscatter coefficient $\sigma^{0}$ and other derived quantities to equivalent quantities, i.e., to an equivalent backscatter coefficient $\sigma_{e}^{0}$, etc. Furthermore, describing the measurement quantity in terms of an equivalent physical object (a sphere) allows one to form a simple mental model of what this quantity means.

The following two sections address the implications of the proposed terminology for measurements and calibration, respectively.

\section{IMPLiCATIONS OF PROPOSED TERMINOLOGY FOR MEASUREMENTS}

Embracing rather than neglecting the frequency and angulardependent radar backscatter is the main mental shift which follows from replacing RCS by equivalent RCS as the radiometric measurement quantity. As long as RCS is used as the measurement quantity, a frequency and angular independent backscattering according to Eq. (1) is (often only inherently) assumed. This is common practice in the SAR literature [1][4]. The assumption of a frequency and angular independent 


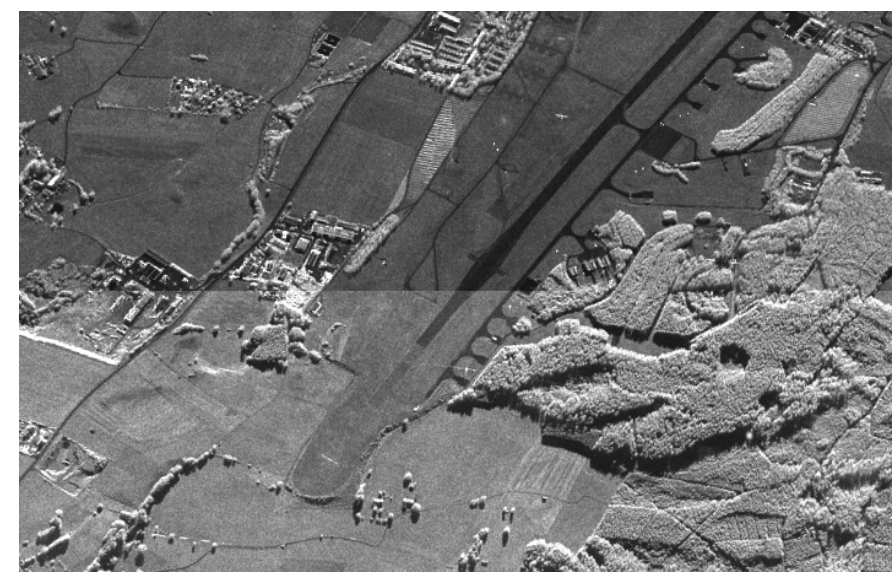

Fig. 1. One scene imaged synchronously by DLR's radiometrically calibrated airborne F-SAR sensor in S-band (upper half) and X-band (lower half), taken in VV polarization on June 8, 2010, at Kaufbeuren, Germany. The desirable difference in backscatter at the two frequency bands is easily discernible. On the other hand, reference point targets with identical equivalent RCS' should appear equally bright in both images.

backscatter introduces a modeling error into the measurement, which in many cases is sufficiently small with respect to the required measurement uncertainty, the utilized range bandwidth and aspect angle range. However, this is not likely to remain the case for upcoming SAR instruments, for which the trend is toward lower radiometric uncertainties combined with higher range bandwidths and longer azimuth integration times for increased resolution. This trend will therefore emphasize this modeling error, which results from the assumption in Eq. (1).

Offered below are two qualitative examples for which a frequency dependent radar backscatter not only exists but is actually the focus for the measurement problem at hand. In these and many other cases, the new terminology of equivalent $R C S$ allows to be precise when reporting measurement results.

a) Multi-spectral SAR images: The frequency dependence of natural targets like agricultural terrain is most apparent when comparing images taken at different frequency bands but otherwise at equivalent time and perspective. The SIRC/X-SAR instrument, which was flown during two missions in 1994, was the first space-borne SAR system capable of operating simultaneously at L-, C-, and X-bands [9]. The fractional bandwidth for each channel was still small at below one percent. Several multi-spectral airborne SAR systems exist among which is the F-SAR system from the German Aerospace Center (DLR) [10]. One exemplary scene, which was imaged simultaneously at two different frequency bands, is shown in Fig. 1, where the fractional bandwidths for the $\mathrm{S}$ and $\mathrm{X}$-band images is $9 \%$ and $8 \%$, respectively. The resulting calibrated images show significant differences in pixel intensities, exemplifying the frequency dependence of the imaged terrain. ${ }^{3}$ This is, of course, expected and desired. After all, the added information content is the actual impetus for building multi-spectral SAR systems like SIR-C/X-SAR and F-SAR.

So what would happen if the same scene was imaged by an

\footnotetext{
${ }^{3}$ The noise-equivalent $\sigma^{0}$ of both channels is well below the measured intensities as not to significantly contribute to the difference in intensities.
}

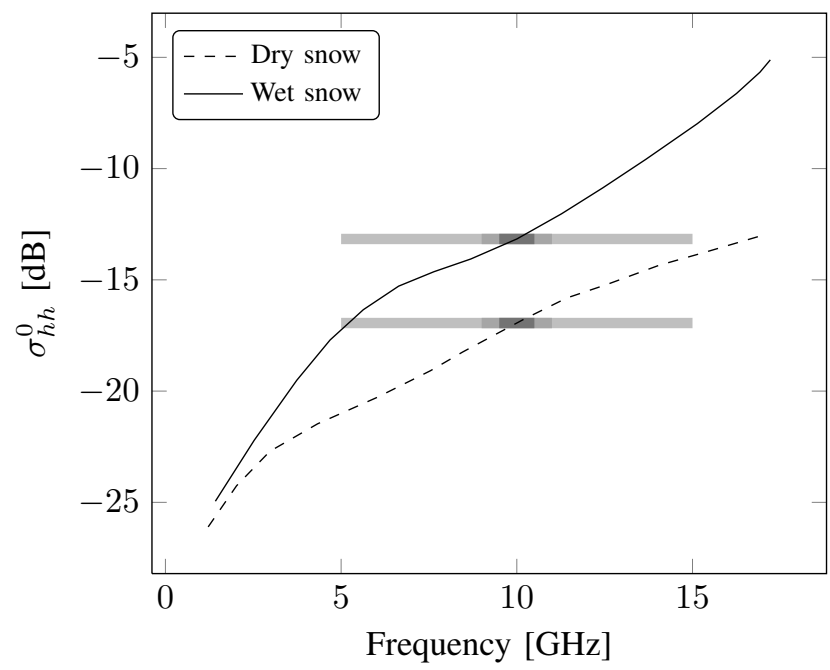

Fig. 2. Frequency dependent backscattering coefficient $\sigma^{0}$ for two exemplary terrain types: dry and wet snow (measured at $\mathrm{HH}$ polarization and an incidence angle of $50^{\circ}$, data from [12]). For illustration purposes, fractional bandwidths of $(10,20,100) \%$ are shown at $10 \mathrm{GHz}$ to emphasize the large frequency dependence within these spectra.

ultra-wideband SAR system? For instance, a fractional bandwidth of $100 \%$ (which is even exceeded by some airborne SAR systems, albeit at the VHF-band [11]) would cover the S- and $\mathrm{X}$-band frequencies in Fig. 1 and all frequencies in between. Hence describing all frequency-dependent terrain radar cross sections within the whole spectrum by a single radar cross section is certainly counter-intuitive and misleading. It would mean that the model uncertainty dominates the radiometric uncertainty budget even if the SAR sensor is well designed and calibrated.

b) RCS over frequency: The frequency dependence of different terrain types was systematically surveyed in [12]. As an example, the frequency dependent backscatter coefficients of dry and wet snow are reproduced in Fig. 2. The backscatter coefficient differs by about $0.4 \mathrm{~dB}$ from its value at $10 \mathrm{GHz}$ within a fractional bandwidth of $10 \%$ for both terrain types, and the difference expectedly increases significantly for larger bandwidths. If this variation is compared to the radiometric accuracy of current (narrower bandwidth) spaceborne SAR instruments, which is typically below $1 \mathrm{~dB}$, it becomes apparent how important the definition of the measurand is. This is especially true in the case of wideband SAR sensors which simply do not see an approximately constant frequency response within their larger range bandwidth. The processed SAR images rather show a weighted RCS average over frequency, a quantity which would be more appropriately termed as equivalent RCS.

The next three quantitative examples show the difference between RCS and equivalent RCS. The way in which the equivalent RCS was derived through simulation for the respective target transfer functions $H_{t}(f, \boldsymbol{\alpha})$ and given SAR systems (defined by their point-spread function $h$ ) is described in detail in [13], where equivalent RCS is called perceived RCS. The approach is based on simulating a point target's integrated pixel intensity and relating it to the integrated pixel intensity 


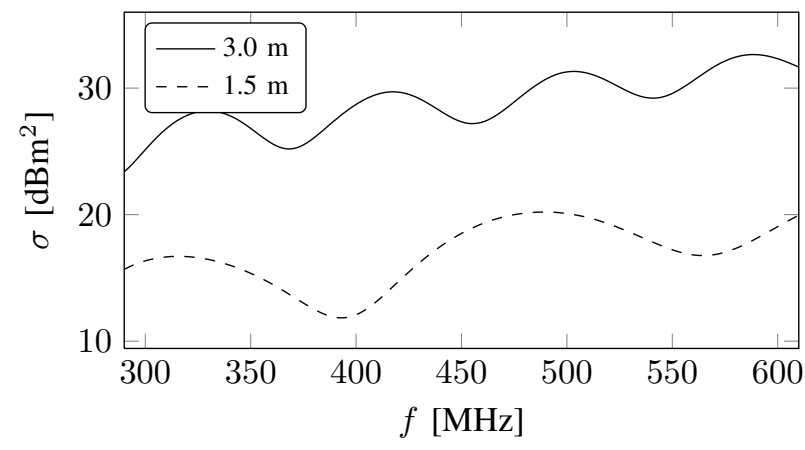

Fig. 3. Peak radar cross section of two small (with respect to the wavelength) trihedral corner reflectors with different inner-leg lengths $l$, simulated with the method of moments.

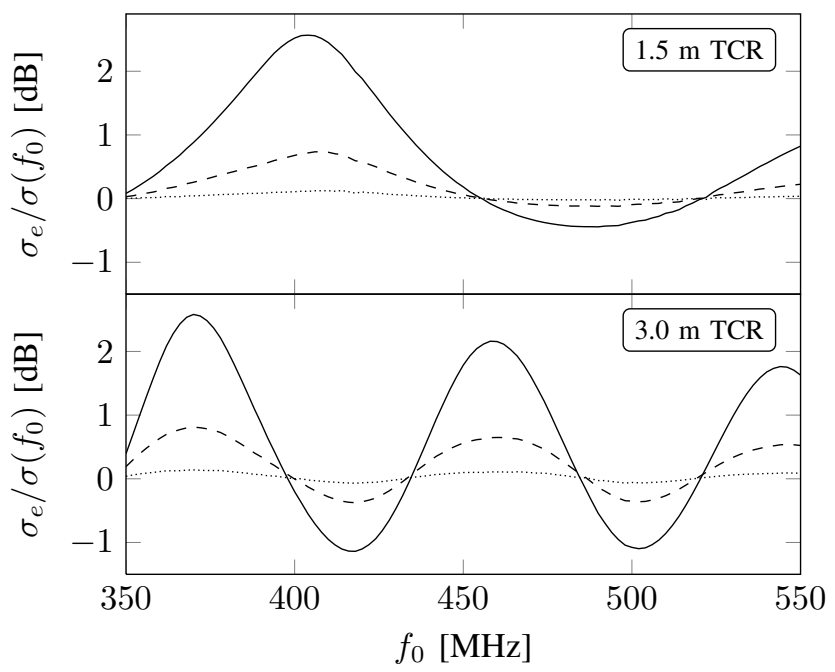

Fig. 4. Difference between equivalent RCS $\sigma_{e}$ and $\operatorname{RCS} \sigma\left(f_{0}\right)$ at the center frequency $f_{0}$ for the two trihedral corner reflectors from Fig. 3 at three bandwidths: $20 \mathrm{MHz}(\cdots \cdots \cdots \cdots), 50 \mathrm{MHz}(---)$, and $100 \mathrm{MHz}(\stackrel{-}{-})$.

of an ideal target, such as a perfectly conducting sphere.

c) Corner reflectors at UHF-band: Corner reflectors are often used as reference targets during radiometric calibration. Their backscatter characteristics therefore need to be accurately known. For reflectors which are large with respect to the wavelength, their frequency-dependent peak RCS is approximated as

$$
\sigma_{\text {corner }}(f)=\frac{4 \pi}{3} \frac{l^{4}}{\lambda^{2}},
$$

where $l$ is the inner-leg length of the corner reflector and $\lambda$ is the wavelength [1]. The RCS at the SAR center frequency lends itself to be the reference RCS.

The simple relationship in Eq. (12) is invalid for reflectors which are small with respect to the wavelength, see Fig. 3. Figure 4 shows the simulated difference between the RCS at the center frequency and the equivalent RCS that would be measured by a F-SAR-like SAR system with varying center frequency and range bandwidth. ${ }^{4}$ No phase or angular variation was assumed for this simulation.

\footnotetext{
${ }^{4}$ Note that the P-band channel of DRL's airborne F-SAR system [10] falls within the plotted range, having a center frequency of $350 \mathrm{MHz}$ and a bandwidth of $100 \mathrm{MHz}$.
}

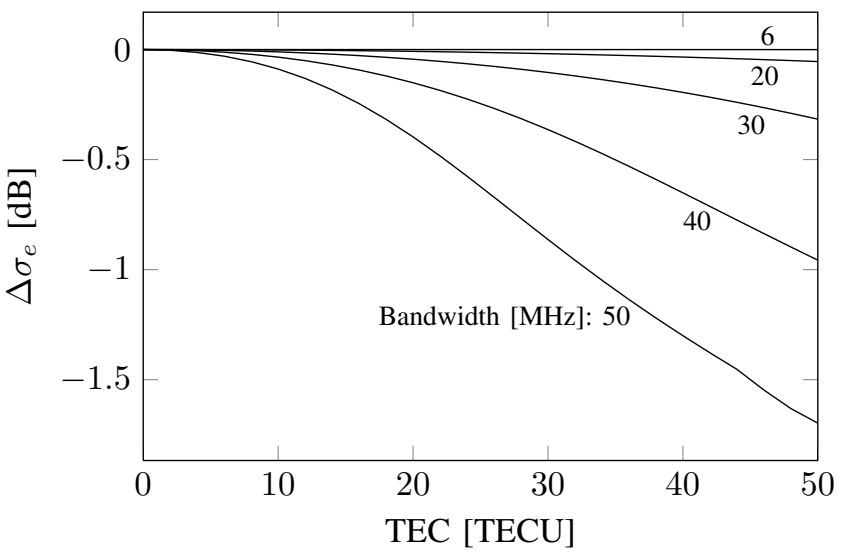

Fig. 5. Effect of phase distortions on the equivalent RCS for a BIOMASSlike system (nominal bandwidth: $6 \mathrm{MHz}$ ) at a center frequency of $350 \mathrm{MHz}$ depending on ionospheric activity. The values are normalized to the equivalent RCS at 0 TECU.

The difference between the equivalent RCS and the RCS at the center frequency, a result of the weighted averaging during processing, ranges from fractions of a decibel to several decibels. This demonstrates the importance of discerning RCS from equivalent RCS, especially for targets used during radiometric calibration.

d) Ionosphere and phase response: Typically, the frequency-dependent amplitude response will have a larger effect on the difference between RCS (at the center frequency) and equivalent RCS than the frequency-dependent phase response. This is because the phase response leads to defocussing only, which is typically well compensated by integrating over $A$ in Eq. (10) [8]. Here, a speculative example shall be given which only considers the phase response of a point target and assumes a constant RCS over frequency and angle.

It has been proposed before to use wideband spaceborne L-band SAR systems for mapping of the ionospheric total electron content (TEC) [14]. Such systems exploit the apparent range delay and defocussing of scatterers on ground depending on ionospheric activity. In contrast to previous examples, the measurand is now a point target including path effects due to the ionosphere.

Neglecting range delays, the quadratic term (leading to defocussing) after Taylor-series expansion of the two-way phase response can be approximated by [14]

$$
\varphi(f)=-\frac{4 \pi}{c_{0}} \frac{40.28}{f_{0}^{3}} T\left(f-f_{0}\right)^{2},
$$

where $f_{0}$ is the center frequency, $c_{0}$ is the speed of light, and $T$ is the TEC in TECU (which seldom exceeds 100 TECU). From this, a transfer function $H_{t}(f)=C \mathrm{e}^{j \varphi(f)}$ according to Eq. (2) with $A(f, \boldsymbol{\alpha})=C=$ const was derived, and the TEC-dependent variation of the equivalent RCS for a BIOMASS-like system simulated, see Fig. 5. Depending on system bandwidth and ionospheric activity, the equivalent RCS deviates by more than $1 \mathrm{~dB}$ from the reference. Note that the RCS, which does not depend on phase (see Eq. (4)), stays constant.

This example stresses again that radiometric measurements in SAR do not only depend on the point target's RCS, but also 


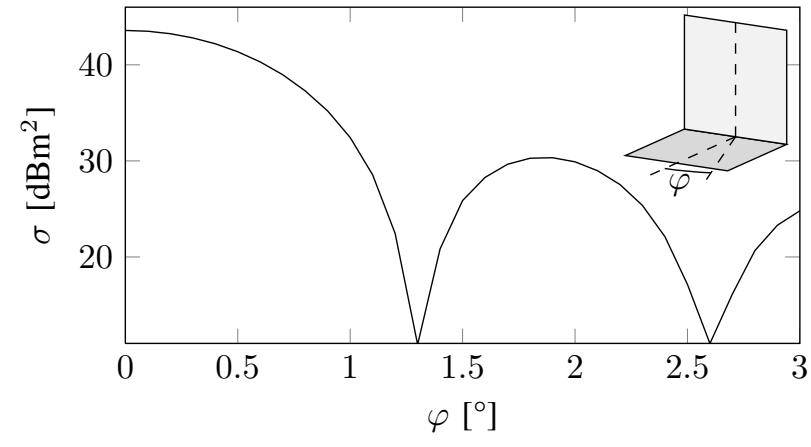

Fig. 6. RCS of a perfectly conducting dihedral corner reflector, where all side lengths are $1 \mathrm{~m}$, at $9.65 \mathrm{GHz}$. The azimuth cut was simulated with the method of moments at an elevation angle of $45^{\circ}$ with respect to the vertical plate.

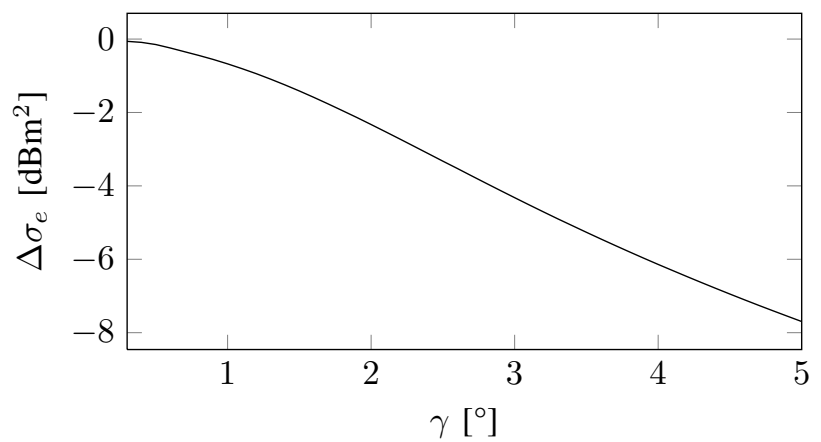

Fig. 7. The normalized equivalent RCS $\Delta \sigma_{e}$ of the dihedral from Fig. 6 as a function of the target exposure time, converted to the SAR beam steering angle $\gamma$, seen by a TerraSAR-X-like system. The values are normalized to the dihedral's peak RCS at $\varphi=0^{\circ}$.

on the target's phase response. It is therefore more appropriate to use the measurement quantity equivalent RCS than RCS to describe pixel intensities.

e) Dihedral corner reflector and angular-dependent $R C S$ : Besides averaging over frequency $f$, a SAR system also averages over a range of aspect angles $\boldsymbol{\alpha}$ during azimuth processing. In case of TerraSAR-X, the angular range $\gamma=\phi_{+}-\phi_{-}$under which a target is seen can be varied from about $0.3^{\circ}$ for a nominal StripMap acquisition to $4.4^{\circ}$ for a high-resolution, experimental Staring Spotlight acquisition [15].

Within this angular range, the RCS of a point target might vary considerably. Figure 6 shows the RCS over azimuth angle for a $1 \mathrm{~m}$ dihedral reflector. Dihedrals with larger dimensions and therefore a higher angular sensitivity are found in urban environments, where many scatterers can be modeled as dihedrals.

Figure 7 shows the large difference between the $1 \mathrm{~m}$ dihedral's peak $\operatorname{RCS}\left(\varphi=0^{\circ}\right)$ and its equivalent RCS for varying azimuth resolutions and therefore angular ranges. This last example emphasizes once more that pixel intensities should not be described by RCS but by their equivalent RCS.

Concluding the examples, it can be said that many natural and man-made targets exhibit a frequency and sometimes angular-dependent RCS. This realization, coupled with the fact that SAR systems record data over certain and sometimes relatively large range bandwidths, demonstrates that RCS is not well suited as the radiometric measurement quantity. Introducing equivalent $R C S$ as the new terminology avoids the ambiguity by distinguishing the body property from the quantity that is seen in a SAR image.

\section{IMPLiCATIONS OF PROPOSED TERMINOLOGY FOR CALIBRATION}

External radiometric calibration is achieved by placing a point target of known backscatter within the imaged scene so that an adequate calibration factor, called $K$ in Eq. (8), can be derived; until now, the reference targets were described by their RCS [1], [2], [16]. With the new understanding that not RCS but equivalent RCS is measured by a SAR instrument, the reference target must now be described by its equivalent RCS and not anymore by its RCS. Two strategies are possible:

1) Build reference targets whose amplitude and phase responses are as constant as technically feasible over the relevant range bandwidth (in which case the target RCS at any frequency within the range bandwidth is close to its equivalent RCS). These targets can thus be described by Eq. (1).

2) Compute the equivalent RCS, the radiometric quantity seen in a SAR image, based on the arbitrary but known transfer function of a reference target according to Eq. (2).

In almost all cases, strategy (1) excludes passive point targets for accurate or wideband SAR systems. Specifically, the RCS of commonly used trihedral corner reflectors is frequency dependent, see example (c) in the previous section. Building active targets (transponders) with a constant frequency response over the complete range bandwidth is, while technically possible, still very challenging. For instance, the frequency dependent transmission of antennas, amplifiers, and filters would need to be compensated by the transponder electronics or otherwise a calibration error would result.

Strategy (2) circumvents the mentioned problems for strategy (1), allowing both active and passive reference targets to be used. The following describes how the calibration procedure needs to be adapted in order to take an arbitrary but known reference target transfer function $H_{t}(f, \boldsymbol{\alpha})$ into account.

Up to now, the calibration coefficient $K$ is simply derived by placing a target of known RCS $\sigma_{e}$ (assuming a sphere-like target according to Eq. (1)) within a scene and determining its integrated pixel intensity $I_{e}$ so that

$$
K=\frac{I_{e}}{\sigma_{e}}
$$

according to Eq. (8). On the other hand, if an arbitrary target according to Eq. (2) is considered, its equivalent RCS $\sigma_{e}$ is not immediately known. It can be derived, however, by processing the raw data with a target-dependent correction point-spread function (PSF), explained in the following.

Assumed to be known through laboratory measurements is the target's complex reflectivity over frequency and incidence angle, expressible as the transfer function $H_{t}(f, \boldsymbol{\alpha})$ or equivalently as the PSF $h_{t}(x, y)$. The target PSF can be normalized 
with respect to a known reference RCS $\sigma_{r}$ at a single reference frequency $f_{r}$ (e.g. the center frequency) and a single reference incidence angle (e.g. the angle at closest approach) $\boldsymbol{\alpha}_{r}$ :

$$
h_{t}(x, y)=\sqrt{\sigma_{r}} h_{t n}(x, y)
$$

with the normalized PSF defined in the Fourier domain by

$$
H_{t n}(f, \boldsymbol{\alpha})=\frac{H_{t}(f, \boldsymbol{\alpha})}{H_{t}\left(f_{r}, \boldsymbol{\alpha}_{r}\right)}
$$

so that

$$
\sqrt{\sigma_{r}}=H_{t}\left(f_{r}, \boldsymbol{\alpha}_{r}\right) .
$$

A correction filter can now be determined which effectively transforms a point target with an arbitrary transfer function into an equivalent ideal (sphere-like) target with a flat response over frequency and angle:

$$
\delta(x, y)=h_{t n}(x, y) * h_{t c}(x, y)
$$

with the target correction PSF $h_{t c}$ defined in the Fourier domain by

$$
H_{t c}(f, \boldsymbol{\alpha})=\frac{1}{H_{t n}(f, \boldsymbol{\alpha})} .
$$

Now, the recorded complex raw data can be processed with a corrected, target-dependent filter $\left(h_{t c} * h\right)$ :

$$
V_{c}(x, y)=\sqrt{K \sigma_{r}}\left[h_{t n} *\left(h_{t c} * h\right)\right](x, y)
$$

In analogy to Eq. (5), an integrated pixel intensity $I_{c}$ can be derived from $V_{c}$. The equivalent RCS $\sigma_{e}$ of the reference target is different from the RCS $\sigma_{r}$ at the reference operating point. The two are related through

$$
\frac{I_{e}}{I_{c}}=\frac{\sigma_{e}}{\sigma_{r}} .
$$

by analogy between Eq. (5) and (15). The ratio $I_{e} / I_{c}$ is the point target and SAR mode dependent correction factor described in [13]. Substituting unknown $\sigma_{e}$ in Eq. (13) with the expression in Eq. (16), the absolute calibration factor is then given as

$$
K=\frac{I_{c}}{\sigma_{r}} .
$$

In conclusion, radiometric measurements can and have to be tied down to a known equivalent RCS by processing the raw data with a filter reversing the frequency and angulardependent reflectivity, $h_{t c} * h$. By using targets with a known equivalent RCS (instead of a known RCS) for calibration, it is ensured that radiometric differences in measurements are due to the properties of the imaged target and not due to the reference target properties.

\section{CONCLUSION}

This paper discussed the problems associated with denoting radar cross section as the radiometric measurement quantity for SAR images. The root cause was identified to be the principal frequency and angular dependence of the target's backscatter. It was proposed to replace RCS with equivalent $R C S$ as the radiometric measurement quantity in SAR images.

This paper has identified two problems with denoting RCS as the radiometric measurement quantity in SAR images:
- The SAR sensor emits pulses covering a certain bandwidth and angular range. However, only one pixel intensity is annotated in the processed SAR image. The pixel intensity is at best proportional to a weighted average of the target RCS within the frequency and aspect angle range.

- RCS is defined as a power ratio; this entails that all phase information is lost. The processing for SAR images, on the other hand, depends on signal magnitude and phase. In other words, a SAR system does not measure RCS and therefore another terminology should be used to describe the measurement quantity. This paper proposed that the quantity equivalent RCS should be used as a replacement. Equivalent $R C S$ was defined to be equal to the radar cross section of a perfectly conducting sphere which would result in an equivalent pixel intensity if the sphere were to replace the measured target.

The implication of the new terminology for measurements was identified to lie in a conscious shift toward recognition of the spectral and angular dependence of target backscatter as a desired feature. It was shown that the difference between RCS and equivalent RCS, prominent especially for wideband and high-resolution SAR systems, can exceed several decibels. On the other hand, in terms of external absolute radiometric calibration, the new terminology asks for a compensation for the frequency and angular dependent backscatter of reference calibration targets by processing raw data with a correction filter during calibration.

By adapting equivalent RCS as the measurement quantity, calibrations and measurement results become truly compatible across current and future narrow and especially wideband, high-resolution, and high-accuracy SAR systems.

\section{ACKNOWLEDGMENTS}

The authors are grateful to Dr. Rolf Scheiber from DLR for providing the F-SAR images and for commenting on an early draft of the article. The quality of the work was also greatly enhanced by the help of Andrew Cook and Jens Fischer. The authors are also grateful to the reviewers for constructive critique and thorough check of the article.

\section{REFERENCES}

[1] A. Freeman, "SAR calibration: An overview," IEEE Transactions on Geoscience and Remote Sensing, vol. 30, no. 6, pp. 1107-1121, Nov. 1992.

[2] J. C. Curlander, Synthetic aperture radar: Systems and signal processing. John Wiley \& Sons, Inc., 1991.

[3] C. Oliver and S. Quegan, Understanding synthetic aperture radar images. SciTech Publishing, 2004.

[4] I. G. Cumming, Digital Processing Of Synthetic Aperture Radar Data: Algorithms And Implementation. Artech House Publishers, 2005.

[5] E. F. Knott, J. F. Shaeffer, and M. T. Tuley, Radar cross section, 2nd ed. Raleigh, NC: SciTech Publishing, 2004.

[6] T. Fritz, "TerraSAR-X ground segment - Level 1b product format specification," Clustert Applied Remote Sensing (CAF), German Aerospace Center (DLR), Tech. Rep. TX-GS-DD-3307, Dec. 2007.

[7] M. Dettwiler, "RADARSAT-2 product format definition," MacDonald, Dettwiler and Associates Ltd., Richmond, B. C., Canada, Tech. Rep. 1/9, Jan. 2011.

[8] A. L. Gray, P. W. Vachon, C. E. Livingstone, and T. I. Lukowski, "Synthetic aperture radar calibration using reference reflectors," IEEE Transactions on Geoscience and Remote Sensing, vol. 28, no. 3, pp. 374-383, May 1990. 
[9] R. L. Jordan, B. L. Huneycutt, and M. Werner, "The SIR-C/X-SAR synthetic aperture radar system," IEEE Transactions on Geoscience and Remote Sensing, vol. 33, no. 4, pp. 829-839, Jul. 1995.

[10] A. Reigber, M. Jager, J. Fischer, R. Horn, R. Scheiber, P. Prats, and A. Nottensteiner, "System status and calibration of the F-SAR airborne SAR instrument," in 2011 IEEE International Geoscience and Remote Sensing Symposium. IEEE, Jul. 2011, pp. 1520-1523.

[11] B. Ulander, Lars M. H.and Flood, P.-O. Frölind, T. Jonsson, A. Gustavsson, J. Rasmusson, G. Stenström, A. Barmettler, and E. Meier, "Bistatic experiment with ultra-wideband VHF-band synthetic-aperture radar," European Conference on Synthetic Aperture Radar, Jun. 2008.

[12] F. T. Ulaby, Handbook of radar scattering statistics for terrain. Artech House, 1989.

[13] B. J. Döring, P. Looser, M. Jirousek, and M. Schwerdt, "Reference target correction based on point target SAR simulation," IEEE Transactions on Geoscience and Remote Sensing, vol. PP, no. 99, Aug. 2011.

[14] F. Meyer, R. Bamler, N. Jakowski, and T. Fritz, "The potential of broadband L-band SAR systems for small scale ionospheric TEC mapping," Fringe 2005 Workshop, Dec. 2005.

[15] P. Prats-Iraola, R. Scheiber, M. Rodríguez-Cassolà, S. Wollstadt, J. Mittermayer, B. Bräutigam, M. Schwerdt, A. Reigber, and A. Moreira, "High Precision SAR Focusing of TerraSAR-X Experimental Staring Spotlight Data," Proceedings of the IEEE International Geoscience and Remote Sensing Symposium, 2012.

[16] M. Schwerdt, B. Bräutigam, M. Bachmann, B. Döring, D. Schrank, and J. Hueso Gonzalez, "Final TerraSAR-X calibration results based on novel efficient methods," IEEE Transactions on Geoscience and Remote Sensing, vol. 48, no. 2, pp. 677-689, Feb. 2010.

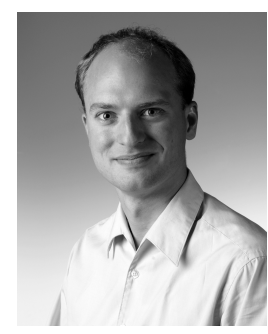

Björn J. Döring received the Dipl.-Ing. degree in electrical engineering from the Technical University Berlin, Germany, in 2005. Since 2006, he has been with the Microwaves and Radar Institute at the German Aerospace Center (DLR), Oberpfaffenhofen, Germany. His research concentrates on satellite SAR calibration with a focus on absolute radiometric calibration. His design and characterization of passive and active calibration targets contribute to precisely calibrated SAR systems like TerraSAR-X, TanDEM$\mathrm{X}$, and GMES Sentinel-1.

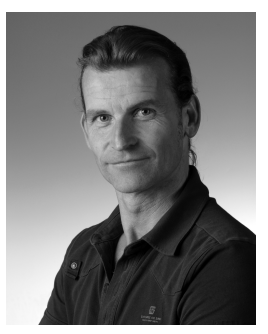

Dr.-Ing. Marco Schwerdt received the Dipl.-Ing degree in electrical engineering and the Dr.-Ing. degree, with a thesis on electrooptical E-field sensors, from the Technical University Berlin, Germany. Since 1998, he has been with the Microwave and Radar Institute at the German Aerospace Center (DLR), Oberpfaffenhofen, Germany, where he is working on SAR calibration methods and performance analysis tools. Since 2000, he has been the head of the Radar Calibration Group performing various radar calibration activities for different SAR missions like XSAR/SRTM or the ScanSAR mode of ASAR/Envisat. He was responsible for the successful calibration of the German TerraSAR-X and TanDEM-X satellites, launched in 2007 and 2010 respectively. Furthermore, as part of the Global Monitoring Environment and Security Program (GMES), he is responsible for developing the overall SAR system calibration and validation plan for ESA's Sentinel-1 mission. Under his leadership, the DLR's comprehensive radar calibration facilities, including novel tools for product quality control and performance analysis, have been maintained and extended. His major research interest includes the development of innovative and efficient calibration methods.

Dr. Schwerdt received the Der Deutsche Gründerfonds award in 1997 for establishing an enterprise of manufacturing electrooptical field sensors under the patronage of the German Federal Minister for Science and Research. 